University of Wollongong

Research Online

Faculty of Engineering - Papers (Archive)

Faculty of Engineering and Information

Sciences

21-11-2005

\title{
Electronic efficiency in nanostructured thermionic and thermoelectric
} devices

Mark F. O'Dwyer

University of Wollongong, markod@uow.edu.au

R. A. Lewis

University of Wollongong, roger@uow.edu.au

C. Zhang

University of Wollongong, czhang@uow.edu.au

T. E. Humphrey

University of California Santa Cruz, USA

Follow this and additional works at: https://ro.uow.edu.au/engpapers

Part of the Engineering Commons

https://ro.uow.edu.au/engpapers/223

\section{Recommended Citation}

O'Dwyer, Mark F.; Lewis, R. A.; Zhang, C.; and Humphrey, T. E.: Electronic efficiency in nanostructured thermionic and thermoelectric devices 2005.

https://ro.uow.edu.au/engpapers/223

Research Online is the open access institutional repository for the University of Wollongong. For further information contact the UOW Library: research-pubs@uow.edu.au 


\title{
Electronic efficiency in nanostructured thermionic and thermoelectric devices
}

\author{
M. F. O’Dwyer,* R. A. Lewis, and C. Zhang \\ School of Engineering Physics and Institute for Superconducting and Electronic Materials, University of Wollongong, \\ Wollongong NSW 2522, Australia \\ T. E. Humphrey \\ School of Physics, University of New South Wales, Sydney NSW 2052, Australia and \\ Baskin School of Engineering, University of California Santa Cruz, Santa Cruz, California 95064-1077, USA \\ (Received 15 June 2005; revised manuscript received 6 September 2005; published 21 November 2005)
}

\begin{abstract}
Advances in solid-state device design now allow the spectrum of transmitted electrons in thermionic and thermoelectric devices to be engineered in ways that were not previously possible. Here we show that the shape of the electron energy spectrum in these devices has a significant impact on their performance. We distinguish between traditional thermionic devices where electron momentum is filtered in the direction of transport only and a second type, in which the electron filtering occurs according to total electron momentum. Such "total momentum filtered" thermionic devices could potentially be implemented in, for example, quantum dot superlattices. It is shown that whilst total momentum filtered thermionic devices may achieve an efficiency equal to the Carnot value, traditional thermionic devices are limited to an efficiency below this. Our second main result is that the electronic efficiency of a device is not only improved by reducing the width of the transmission filter as has previously been shown, but also strongly depends on whether the transmission probability rises sharply from zero to full transmission. The benefit of increasing efficiency through a sharply rising transmission probability is that it can be achieved without sacrificing device power, in contrast to the use of a narrow transmission filter which can greatly reduce power. We show that devices that have a sharply rising transmission probability significantly outperform those that do not and that such transmission probabilities may be achieved with practical single and multibarrier devices. We discuss how the shape of the electron energy spectrum will also have an effect on the electronic efficiency of thermoelectric devices due to mathematical equivalences in the ballistic and diffusive formalisms. Finally, we present an experimental measure that might be used to provide an indication of the nature of the electron energy spectrum and the electronic efficiency of a ballistic device.
\end{abstract}

DOI: 10.1103/PhysRevB.72.205330

PACS number(s): 73.63.-b, 73.23.Ad, 72.15.Jf, 79.40.+z

\section{INTRODUCTION}

Traditional vacuum thermionic power generators ${ }^{1-3}$ with macroscopic gaps between emitter and collector plates are limited to very high temperature applications $\left(T_{H}\right.$ $>1000 \mathrm{~K})$. Refrigeration using such devices, as first suggested by Mahan, ${ }^{4}$ is also limited to high temperatures due to a lack of suitable materials with work functions below $\sim 0.3 \mathrm{eV}$.

Nanostructures are currently being investigated in an attempt to develop thermionic devices that can refrigerate or generate power at lower temperatures. The potential for achieving lower barrier heights via the use of semiconductor heterostructures was pointed out by Shakouri and Bowers, 5,6 with Mahan et $a l^{7,8}$ suggesting multilayers as a means of reducing the phonon heat leaks inherent in the use of solidstate rather than vacuum devices. Successful solid-state thermionic cooling of up to a few degrees has been reported. ${ }^{9-12}$

Another direction is the use of nanometer gaps between the emitter and the collector to lower the work function via quantum tunneling, ${ }^{13,14}$ with Hishinuma et al. reporting cooling of about a millidegrees Kelvin in such a system. Cooling by field emission from carbon nanotubes and other nanostructures has also been proposed..$^{15,16}$

In thermoelectrics, nanostructured devices may offer the possibility of substantially increasing the thermoelectric fig- ure of merit, $Z T$, over that of traditional bulk bismuth telluride based devices $(Z T \approx 1)$ due to enhanced electron transport and phonon blocking properties. Hicks and Dresselhaus have predicted that $Z T$ can be enhanced using quantum-well superlattices ${ }^{17}$ and quantum wires. ${ }^{18}$ Venkatasubramanian et al. have reported the highest $Z T$ to date, $Z T \sim 2.4$, using a $p$-type $\mathrm{Bi}_{2} \mathrm{Te}_{3} / \mathrm{Sb}_{2} \mathrm{Te}_{3}$ superlattice. ${ }^{19}$ Other methods used and suggested for the enhancement of the figure of merit include the use of quantum-dot superlattices, ${ }^{20,21}$ superlatices with a nonconservation of lateral momentum, ${ }^{22,23}$ inhomogeneous doping, ${ }^{24,25}$ and nanotubes. ${ }^{26}$

Many of these approaches offer the possibility of engineering the electron energy spectrum (the number of electrons transmitted through the device as a function of energy) in a way that was not possible in traditional vacuum thermionics or bulk thermoelectrics. In light of the design freedom offered by nanostructures, it is useful to re-examine the impact of the electron energy spectrum upon what has been called the "electronic efficiency" of thermionic devices, ${ }^{27}$ defined as the efficiency associated with strictly electronic processes under ideal conditions of particle transport.

Improvements in electronic efficiency due to better device design that can be achieved without lowering the power will translate into an improvement in the operating efficiency of practical thermionic devices where nonideal effects, such as phonon and radiative leaks, as well as contact and lead re- 
(a)

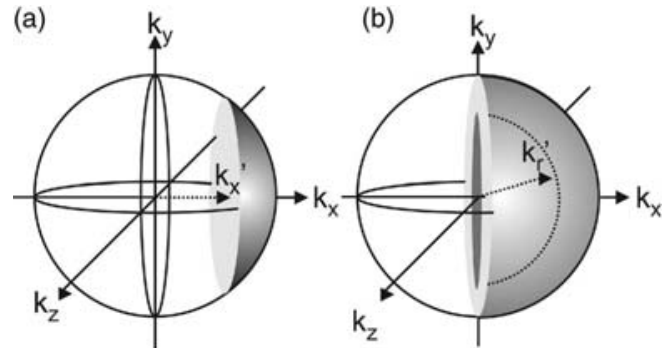

FIG. 1. Fermi spheres indicating electrons transmitted by (a) a $k_{x}$ filtered device and (b) a $k_{r}$ filtered device.

sistances, are important. To achieve high overall efficiency in practical devices it is important to design devices that not only achieve low thermal conductivity, but high electronic efficiency at finite power as well.

In this paper we analyze in detail the dependence of the electronic efficiency of thermionic power generators and refrigerators upon the details of the energy spectrum of electrons transmitted ballistically between the emitter and collector. The term energy filtering is often used to indicate a restriction of electron flux to all those electrons above a certain energy. Here the term energy filtering will be used in a more general sense to indicate any arbitrary restriction on the energy spectrum of transmitted electrons. We examine two idealized models of thermionic nanodevices. In the first, energy (or more precisely, momentum) filtering of electrons occurs in the direction of transport only. This model, which we will denote as a " $k_{x}$-filtered thermionic device," is applicable to single-barrier or multibarrier (superlattice) solidstate devices, which are translationally invariant in the directions perpendicular to transport. In the second model, which we will denote as a $k_{r}$-filtered thermionic device, energy filtering of the total energy of electrons is assumed to be possible. This model is applicable to vacuum emission from nanostructures such as carbon nanotubes and solid-state devices in which there is periodic modulation of the potential in all three dimensions (such as quantum dot superlattices), or superlattices in which there is nonconservation of electron momentum in directions perpendicular to transport. ${ }^{22,23}$ There are many other physical systems in which the electron emission process is dependent on the total energy of electrons and therefore might be mathematically characterized as a $k_{r}$ system, such as electron emission from electrons. Figure 1 shows geometrically the range of electrons transmitted in idealized $k_{x}$ and $k_{r}$ type devices in momentum space.

In thermoelectric devices, the presence of a band gap in different crystallographic directions ensures that electrons contributing to the current have a certain minimum value of momentum in all three dimensions. The close relationship between thermionic and thermoelectric devices has been analyzed by a number of authors. ${ }^{8,28-30}$ In this paper, this comparison is extended to similarities between the dependence of the electronic efficiencies of these devices on the details of the electron energy spectrum.

\section{TRANSPORT THEORY}

\section{Ballistic transport theory}

A thermionic device consists of two electron reservoirs at different temperatures and electrochemical potentials, sepa- rated by a barrier, or series of barriers, that limit the flow of electrons between them to a certain energy range. Whether the device operates as a power generator, pumping highenergy electrons from the hot to the cold reservoir against the electrical potential difference, or as a refrigerator, removing high-energy electrons from the cold reservoir, depends upon the relative magnitudes of the opposing temperature and electrochemical potentials.

The energy dependent transmission probability, $\zeta$, gives the probability of an electron in a reservoir successfully traveling to the opposite reservoir. In a $k_{r}$-filtered thermionic device, where the transmission probability is a function of the total electron energy, $E=\hbar^{2} \mathbf{k}^{2} / 2 m^{*}$, the net electrical current density flowing from the cold to the hot reservoir is

$$
J_{r}=q \int_{0}^{\infty}\left[n_{r}^{C}-n_{r}^{H}\right] \zeta(E) d E
$$

where $q=-1.602 \times 10^{-19} \mathrm{C}$ is the charge of an electron and

$$
n_{r}^{C / H}=\frac{m^{*} E}{2 \pi^{2} \hbar^{3}} f\left(E, \mu_{C / H}, T_{C / H}\right)
$$

is the number of electrons with total energy $E$ arriving at the three-dimensional reservoir interface per unit area per unit time and

$$
f\left(E, \mu_{C / H}, T_{C / H}\right)=\left[1+\exp \left(\frac{E-\mu_{C / H}}{k_{B} T_{C / H}}\right)\right]^{-1}
$$

is the Fermi-Dirac distribution function in the cold/hot reservoir with electrochemical potential $\mu_{C / H}$ and temperature $T_{C / H}$. We have assumed that electron velocity is determined by the reservoirs. A more detailed theory would be required to account for any velocity changes due to the device structure.

One may calculate the heat flux out of the hot and cold reservoirs by noting that an electron leaving or entering the cold/hot reservoir will remove or add respectively an amount of heat equal to the difference between the energy of the electron and the average energy of electrons in the reservoir, that is $E-\mu_{C / H}$. Introducing this factor inside the integral for number current we may obtain expressions for the net heat flux out of the cold/hot reservoir as

$$
\dot{Q}_{r}^{C / H}=\mp \int_{0}^{\infty}\left(E-\mu_{C / H}\right)\left[n_{r}^{C}-n_{r}^{H}\right] \zeta(E) d E .
$$

In a $k_{x}$-filtered device the transmission probability is a function of what may be loosely defined as the "kinetic energy of electrons in the $x$ direction," $E_{x}=\hbar^{2} k_{x}^{2} / 2 m^{*}$. It is therefore convenient in this case to write the electrical and heat currents in terms of $E_{x}$ (Ref. 31)

$$
J_{x}=q \int_{0}^{\infty}\left[n_{x}^{C}-n_{x}^{H}\right] \zeta\left(E_{x}\right) d E,
$$

where 


$$
n_{x}^{C / H}=\frac{m^{*} k_{B} T_{C / H}}{2 \pi^{2} \hbar^{3}} \log \left[1+\exp \left(-\frac{E_{x}-\mu_{C / H}}{k_{B} T_{C / H}}\right)\right]
$$

is the number of electrons with kinetic energy in the $x$ direction $E_{x}$ arriving at the reservoir interface per unit area per unit time.

In a $k_{x}$-filtered device, the average heat removed from the cold/hot reservoir when an electron with energy in the $x$ direction $E_{x}$ leaves, $E_{x}+k_{B} T_{C / H}-\mu_{C / H}$ (assuming MaxwellBoltzmann statistics), is not the same as that added when an electron with energy in the $x$ direction $E_{x}$ arrives, $E_{x}$ $+k_{B} T_{H / C}-\mu_{C / H}$. This difference is due to the fact that, while the barrier system filters electrons according to their momentum in the direction of transport, their momenta in the other two dimensions may take any value, contributing on average an extra $k_{B} T_{C / H}$ to the energy of electrons emitted from the cold/hot reservoir. The heat flux out of the cold/hot reservoir in a $k_{x}$ filtered device is therefore given by

$$
\begin{aligned}
\dot{Q}_{x}^{C / H}= & \mp \int_{0}^{\infty}\left[\left(E_{x}+k_{B} T_{C / H}-\mu_{C / H}\right) n_{x}^{C}\right. \\
& \left.-\left(E_{x}+k_{B} T_{H / C}-\mu_{C / H}\right) n_{x}^{H}\right] \zeta\left(E_{x}\right) d E .
\end{aligned}
$$

It may be noted that many cryogenic ballistic refrigerators such as normal-insulating-semiconductor (NIS) junction devices $^{32-34}$ and quantum dot refrigerators ${ }^{35}$ utilize either two- or one-dimensional reservoirs where the difference between $k_{x}$ and $k_{r}$ filtered devices is less dramatic or nonexistent.

The electronic efficiency as a power generator and coefficient of performance (COP) as a refrigerator for both $k_{x}$ and $k_{r}$ filtered devices are given by

$$
\eta^{P G}=V J / \dot{Q}^{H}
$$

and

$$
\eta^{R}=\dot{Q}^{C} / V J,
$$

respectively, where $V=\left(\mu_{C}-\mu_{H}\right) / q$.

\section{A. Diffusive transport theory}

Thermoelectric devices are generally differentiated from thermionic devices according to whether electron transport is diffusive or ballistic. ${ }^{8}$ There is, however, little to distinguish the underlying thermodynamics of the two types of device, with both achieving reversibility under the same conditions ${ }^{25}$ and both being governed by the same "materials parameter." $29,30,37$

Under the relaxation-time approximation the electric current in a thermoelectric device may be calculated using the Boltzmann transport equation as

$$
J^{d}=\iiint q D_{l}\left[v_{x}^{l}\right]^{2} \tau \frac{d f}{d x} d \mathbf{k},
$$

where $D_{l}$ is the local density of states (DOS), $\tau=\tau_{0} E^{r}$ is the relaxation time, and $v_{x}^{l}=(1 / \hbar)\left[\partial E\left(k_{x}\right) / \partial k_{x}\right]$ is the velocity in the direction of transport. The electron energy spectrum in a diffusive device is thus determined by $D_{l}\left[v_{x}^{l}\right]^{2} \tau[d f / d x]$.
The transport equation for ballistic devices, where the mean free path of an electron between collisions is greater than the width of the barrier, or system of barriers, may be written similarly as

$$
J^{b}=\iiint q D_{r} \zeta v_{x}^{r} \Delta f d \mathbf{k},
$$

where $D_{r}=1 /(2 \pi)^{d}$ is the DOS in $k$-space in the reservoirs where $d$ is the dimensionality of the reservoirs, and $\Delta f=f_{C}$ $-f_{H}$ is the difference between the distribution functions in the cold and hot reservoirs. The electron energy spectrum in a ballistic device is therefore determined by $D_{r} v_{x}^{r} \zeta \Delta f$.

We expect that Eqs. (10) and (11) should yield the same results for devices of width close to the electron mean free path. If we take the energy dependence of the relaxation time to be $r=-1 / 2$, which is appropriate when scattering is dominated by acoustic phonons, the mean free path in the direction of transport will be independent of energy and given by $\lambda \equiv v_{x} \tau{ }^{30}$ For a small piece of thermoelectric material of length approximately equal to the electron mean free path $d f / d x \approx \Delta f / \lambda$. Equation (10) then reduces to ${ }^{30}$

$$
J^{d}=\iiint q D_{l} v_{x}^{l} \Delta f d \mathbf{k}
$$

and is of the same form as that of the ballistic transport equation, Eq. (11). Thus, the term $D_{l} v_{x}^{l}$ in the diffusive formalism plays the same role as $D_{r} v_{x}^{r} \zeta$ in the ballistic formalism. We therefore expect the dependencies of the electron energy spectrum in both thermionic and thermoelectric devices to be similar.

Due to the equivalence of the diffusive and ballistic formalisms in this regime, the intensive efficiency across a small section of thermoelectric material ${ }^{38,39}$ and the electronic efficiency/COP of a ballistic device are given by Eqs. (8) and (9), respectively.

\section{REVERSIBLE ELECTRON TRANSPORT}

To achieve reversibility in a thermionic or thermoelectric device, electrons must flow only at energies where the Fermi occupation of states, Eq. (3), is constant. ${ }^{25,36}$ Assuming a finite temperature difference at each end of the device, there are two different quasi-static limits in which this requirement is satisfied. The first way is to restrict the flow of electrons to those with energies approaching infinity where the occupation of states tends to zero. This may be achieved, for instance, with an intrinsic semiconductor where the band gap approaches infinity in a thermoelectric device or an infinitely high barrier system in a thermionic device.

The second way to achieve reversibility in a thermionic or thermoelectric device, which we refer to as energy-specific equilibrium, is to allow electrons to flow only at a single energy where the Fermi occupation of states throughout the device is the same ${ }^{36}$

$$
E_{0}=\frac{\mu(T+\delta T)-(\mu-\delta \mu) T}{\delta T},
$$

where $\delta T$ and $\delta \mu$ are the temperature and electrochemical potential changes, respectively, over a distance $\delta x$ in the de- 
vice. At this energy, the effect, or, in the language of irreversible thermodynamics, the "affinity," 40 of the opposing temperature and electrochemical potential gradients upon electrons exactly cancels and transport occurs reversibly. This is also the energy at which the energy-resolved current changes sign, that is, for electrons with energies less than $E_{0}$ the net current flows from the cold to hot reservoir and for energies greater than $E_{0}$ net current flows from the hot to cold reservoir. Transport of electrons with energy close to a single value only might be achieved using resonant tunneling in a superlattice or quantum dot superlattice. For a thermionic device, the ballistic transmission energy is determined substituting the cold and hot reservoir temperatures and electrochemical potentials into Eq. (13). Here we shall denote a filtering system that transmits only a single energy of electrons between the reservoirs, be that the single total energy for a $k_{r}$ device or a single $x$ energy for a $k_{x}$ device, as an "ideal filter". For a ballistic device this may be expressed as a transmission probability function as

$$
\zeta(E)=\left\{\begin{array}{cc}
1 & E=E^{\prime} \\
0 & \text { elsewhere }
\end{array}\right.
$$

where $E_{x}$ would be substituted for $E$ in a $k_{x}$ system.

In a thermoelectric device, inhomogeneous doping or a graded band structure is required so that Eq. (13) may be satisfied at every point in the device for a particular temperature gradient.

\section{ELECTRONIC EFFICIENCY WITH IDEAL FILTERING}

Under ideal filtering, as defined in the previous section, Eqs. (8) and (9) for the $k_{r}$ device reduce to

$$
\eta_{r}^{P G}=\frac{\mu_{C}-\mu_{H}}{E_{0}-\mu_{C}}
$$

for power generation $\left(E^{\prime}>E_{0}\right)$ and

$$
\eta_{r}^{R}=\frac{E_{0}-\mu_{C}}{\mu_{C}-\mu_{H}}
$$

for refrigeration $\left(E^{\prime}<E_{0}\right)$. The efficiency and COP of ideally filtered $k_{x}$ and $k_{r}$ systems are plotted in Fig. 2 relative to the Carnot values. The energy axis for the $k_{x}$ device shown in Fig. 2 is the average total cold reservoir energy, $E_{x}+k_{B} T_{C}$.

When the filtering energy is $E_{0}$, reversibility and the Carnot efficiency are achieved for the $k_{r}$ device as shown in Fig. 2. For all values of total energy shown in Fig. 2, the $k_{r}$ device outperforms the $k_{x}$ device. Importantly, unlike the $k_{r}$ device, the $k_{x}$ filtered thermionic device does not reach the Carnot efficiency for arbitrary electrochemical potentials and finite barrier heights. The reason for this is that although momentum in the $x$ direction is restricted to a single value, the momentum in the $y$ and $z$ directions may take any value, meaning that the total energy spectrum has a finite width and reversibility is not achieved. The distributed nature of total electron energies for a $k_{x}$ device, even with a narrow filter, is shown in Fig. 3. For $k_{x}$-filtered power generators, this upper bound upon the electronic efficiency can be obtained analyti-

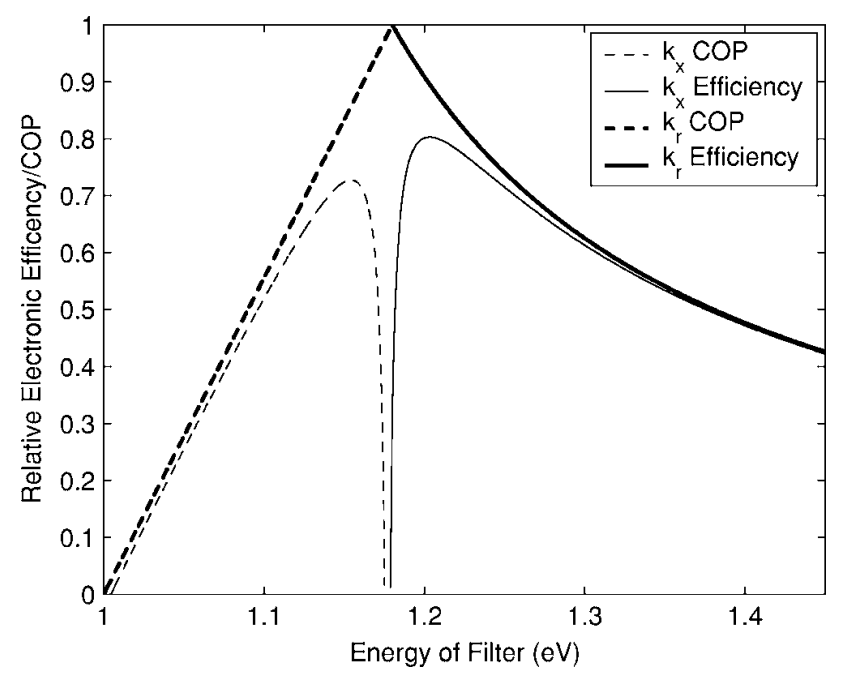

FIG. 2. Relative efficiency and COP of ideally filtered $k_{r}$ and $k_{x}$ devices versus the energy of the ideal filter. The $k_{x}$ curves are plotted against the total average energy of electrons leaving the cold reservoir, $E_{x}+k_{B} T_{C} . T_{H}=300 \mathrm{~K}, T_{C}=270 \mathrm{~K}, \mu_{H}=0.98 \mathrm{eV}$, and $\mu_{C}$ $=1.00 \mathrm{eV}$.

cally in the limit that $\left(\mu_{C}-\mu_{H}\right) / k_{B}\left(T_{H}-T_{C}\right) \gg 1$, in which case maximum efficiency is obtained when $E^{\prime}=E_{0}$, where

$$
\eta_{x}^{P G}=\eta_{C}\left[1+\eta_{C}\left(k_{B} T_{H}+k_{B} T_{C}\right) / q V\right]^{-1},
$$

where $\eta_{C}$ is the Carnot efficiency. This always gives an efficiency less than the Carnot value. This constitutes the first

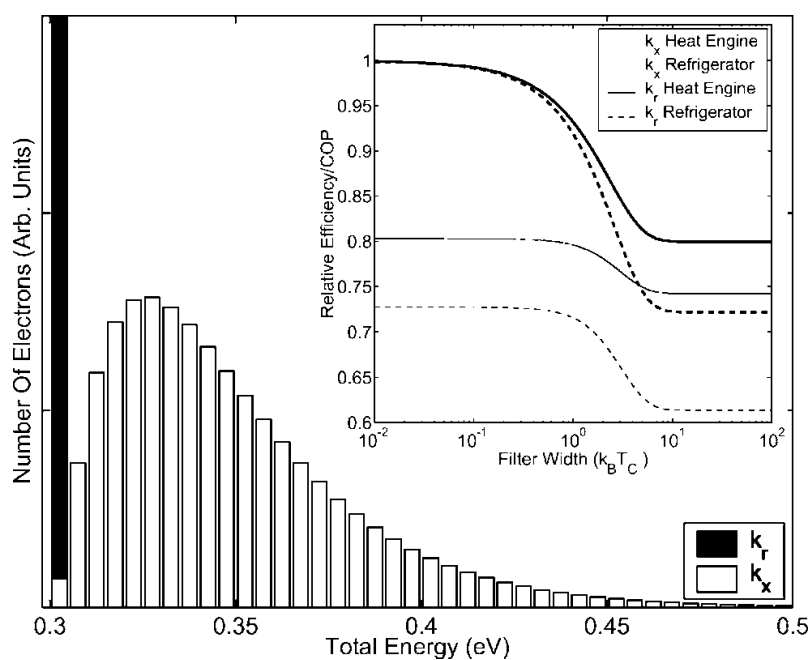

FIG. 3. The total energy distributions for electrons leaving a reservoir in $k_{x}$ and $k_{r}$ filtered thermionic devices with a filter of $5 \mathrm{meV}$ at $0.3 \mathrm{eV}$. The vertical axis has been cut off for clarity of the details in the $k_{x}$ system values. The number of electrons transmitted in the $k_{r}$ system is approximately 13 times that of the $k_{x}$ system for these parameters. $T=270 \mathrm{~K}$ and $\mu=0.1 \mathrm{eV}$. (Inset) The maximum relative efficiency and COP as a fraction of the Carnot limit for $k_{r}$ and $k_{x}$ filtered thermionic devices vs the width of the filter from $0.01 k_{B} T_{C}$ to $100 k_{B} T_{C} . T_{H}=300 \mathrm{~K}, T_{C}=270 \mathrm{~K}, \mu_{H}=0.98 \mathrm{eV}$, and $\mu_{C}=1.00 \mathrm{eV}$. 
main result of the paper, that for finite barrier heights and electrochemical potential differences, $k_{x}$ filtered thermionic devices are limited to a maximum electronic efficiency less than the Carnot limit. This means that from the point of view of maximizing electronic efficiency, $k_{r}$ devices are inherently superior to $k_{x}$ devices.

\section{ELECTRONIC EFFICIENCY WITH NONIDEAL FILTERING}

The filters considered in Sec. IV represent an idealized theoretical limit. We now extend our analysis to nonideal filters, considering filters which transmit a finite range of electrons, and then analyze the effect a gradual rise in the transmission probability has on electronic efficiency.

\section{A. Effect of finite filter width}

A filter of finite width corresponds to a transmission probability of

$$
\zeta(E)=\left\{\begin{array}{cc}
1 & E^{\prime}<E<E^{\prime}+\Delta E \\
0 & \text { elsewhere }
\end{array}\right.
$$

for a $k_{r}$ system, and where $E_{x}$ would be substituted for $E$ for a $k_{x}$ system. Such a filter might be used, for example, to approximate a transmission miniband in a superlattice device and $E^{\prime}$ would be positioned by, say, changing the barrier height relative to the electronchemical potential. For each filter width examined numerically, the starting energy of the filter, $E^{\prime}$, was tuned to find the maximum electronic efficiency/COP for that width and the results are plotted in Fig. 3 (inset). This shows that we do not require an ideal filter to achieve an efficiency/COP very close to the maximum value as seen in the low energy plateau in all curves. Narrow filters with widths in these ranges are achievable using practical semiconductor devices. As the filter widths increase beyond these values the efficiency/COP drops and then plateaus again at a final value where the distribution function approaches zero at higher energies.

Figure 4 shows the energy spectrum of the net electric current transmitted from the hot to cold reservoir for a $0.3 \mathrm{eV}$ wide filter. Results are normalized by the net number of electrons with total energy greater than the Fermi energy available to flow between reservoirs. This illustrates the energy range, with respect to the $x$ and total energies for $k_{x}$ and $k_{r}$ devices, respectively, of the filter for each system when tuned for maximum electronic efficiency/COP. Figure 4 shows that there are more electrons being transmitted for the $k_{r}$ system than with the $k_{x}$ system, an effect previously pointed out by Vashaee and Shakouri, ${ }^{22,23}$ which results in greater power in a $k_{r}$ device. The calculations presented here show that the difference in the energy spread of electrons in $k_{x}$ and $k_{r}$ filtered devices also gives an increase in the electronic efficiency for $k_{r}$ devices due to a greater concentration of electrons with energies around $E_{0}$. For both refrigeration and power generation, the filters will be positioned such that electrons with energy $E_{0}$ are included. Since when $E>E_{0}$ the net energy-resolved current produces power, the lower edge of the $k_{r}$ power generator filter will always be at $E_{0}$. Energy-
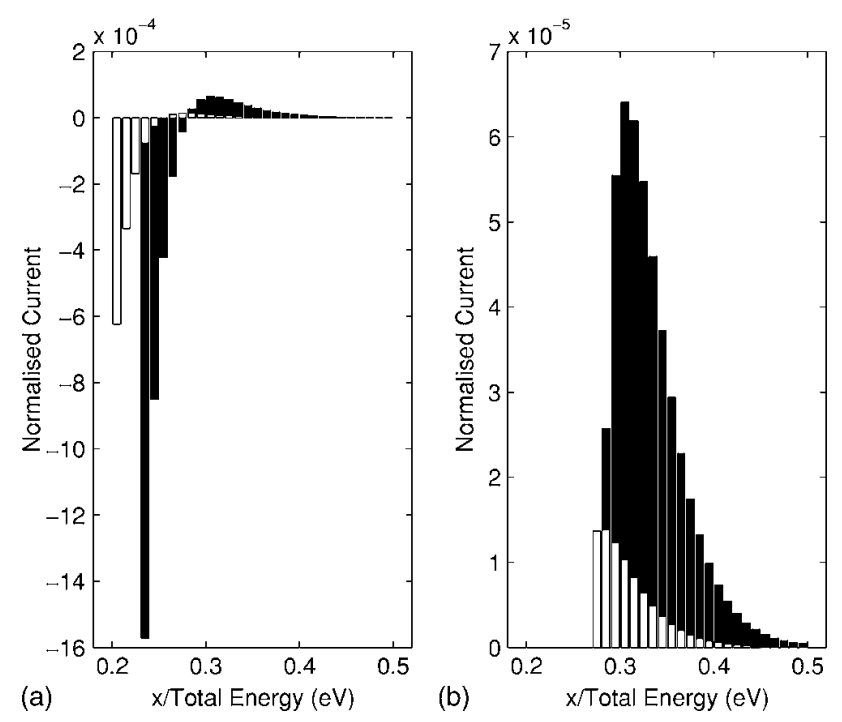

FIG. 4. The normalized electron energy spectrum for net current from the hot to cold reservoir. The filter width is $0.3 \mathrm{eV}$ and is positioned to achieve maximum efficiency/COP in each system for (a) refrigeration and (b) power generation in $k_{r}$ (black) and $k_{x}$ (white) systems. $T_{C}=270 \mathrm{~K}, T_{H}=300 \mathrm{~K}$, and $\mu_{C}=0.1 \mathrm{eV}, \mu_{H}$ $=0.08 \mathrm{eV}$.

resolved current in the energy range $\mu_{C}<E<E_{0}$ refrigerates the cold reservoir and the lower edge of the filter is therefore shifted to this region in Fig. 4(a). Since there are more electrons at higher energies, however, current flow in the region $E>E_{0}$ generates power and a tradeoff occurs when positioning the filter for maximum COP. In both cases, the lower edge of the $k_{x}$ filter is shifted to lower energy due to the additional total energy contribution by the unfiltered degrees of freedom. It should be noted that electrons transmitted with energy below $\mu_{C}$ both absorb power and heat the cold reservoir.

\section{B. Transmission probabilities with finite slopes}

Thus far we have considered only the case where there is a sharp transition from zero to full transmission of electrons. In this section we consider the effect upon the electronic efficiency of a gradual transition, that more closely resembles the shape of the transmission probability in practical devices. We begin by using two convenient "artificial" transmission probabilities, the slope of which can be easily varied. The first, a Gaussian peak that might approximate the transmission probability of a resonance, is given by $\zeta(E)$ $=\exp \left[-\left(E-E_{c}\right)^{2} / w\right]$, where $E_{c}$ defines the center energy of the peak and $w$ is a width parameter that is used to vary the sharpness of the slope. $E_{x}$ would be substituted for $E$ for a $k_{x}$ device.

The second artificial transmission probability considered is a "half-Gaussian" intended to approximate the transmission probability of a single barrier of finite width. This is given by the Gaussian equation for $E \leqslant E_{C}$ and is equal to one for $E>E_{C}$. The sharpness of the Gaussian and halfGaussian transmission probabilities were varied between $w$ 

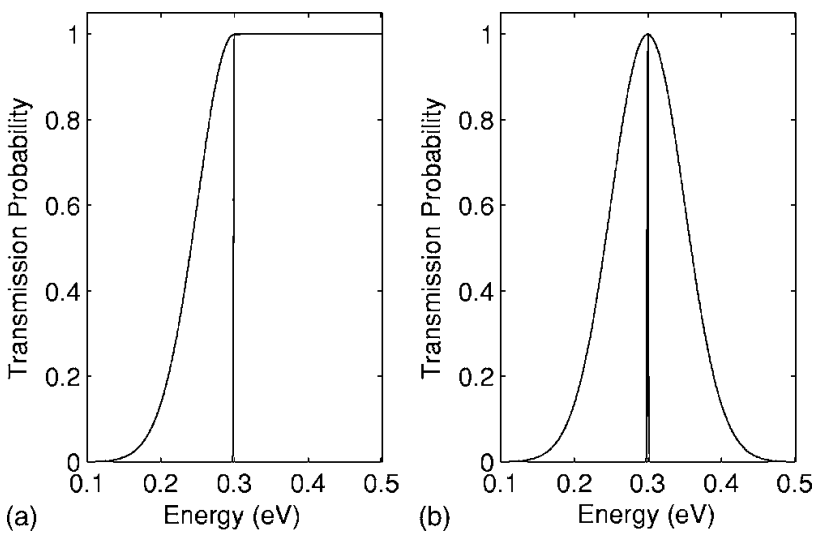

FIG. 5. Artificial transmission probabilities generated using a (a) Gaussian function and (b) a half-Gaussian function. The two extremes of width parameter are shown, $w=0.1$ (slowly rising) and $w=0.000,01$ (sharply rising).

$=10^{-5}$, corresponding to an ideal filter or perfectly sharp single barrier transmission probability, and $w=0.1$. The transmission probabilities associated with these extreme values are shown in Figs. 5(a) and 5(b). The system bias voltage was tuned for each transmission probability for maximum electronic efficiency/COP. Figure 6(a) shows the COPs associated with a room temperature refrigerator and Fig. 6(b) shows the electronic efficiencies of a heat engine operating at higher temperature.

Since all electrons of energy other than $E_{0}$ reduce the electronic efficiency, we expect the sharpest peak in Figs. 6(a) and 6(b) to yield the highest efficiency/COP, and this is confirmed by the numerical results. The most interesting result, however, is that the electronic efficiency of the halfGaussian transmission probability is very strongly dependent upon how sharply the transmission rises from zero to unity.
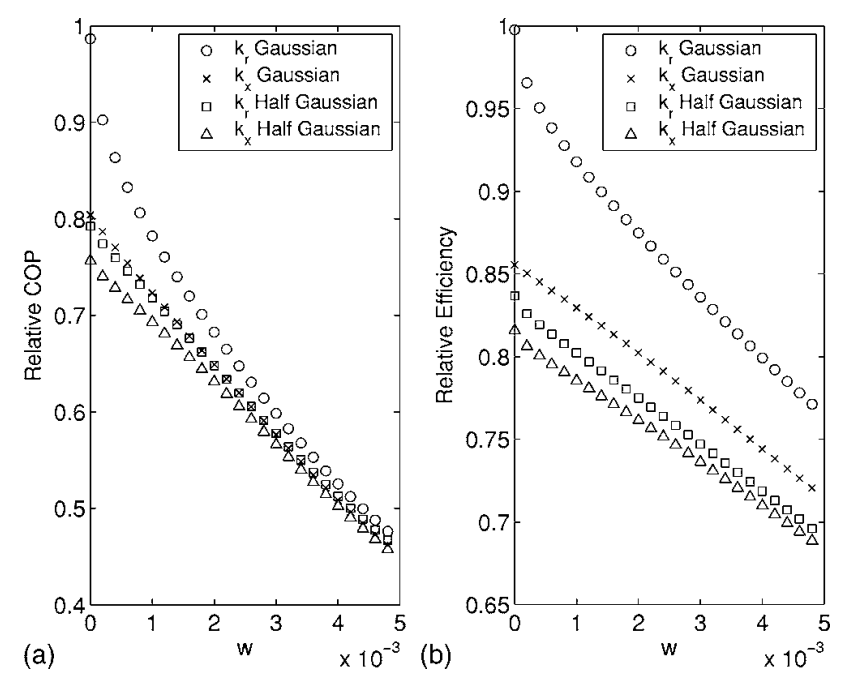

FIG. 6. The efficiency/COP relative to the Carnot value of a $k_{r}$ Gaussian, $k_{x}$ Gaussian, $k_{r}$ half-Gaussian, and $k_{x}$ half-Gaussian for (a) refrigerator systems $\left(T_{H}=300 \mathrm{~K}\right.$ and $\left.T_{C}=270 \mathrm{~K}\right)$ and (b) heat engine systems $\left(T_{H}=900 \mathrm{~K}\right.$ and $\left.T_{C}=300 \mathrm{~K}\right)$ vs width parameter with $\mu_{C}=0.1 \mathrm{eV}$.
A smooth rise in the transmission probability lowers the electronic efficiency for the same physical reason that a $k_{x}$ filtered device has a lower electronic efficiency than a $k_{r}$ filtered device. Whenever the energy spectrum of transmitted electrons rises slowly to its peak value there is an efficiency lowering tradeoff that occurs between transmitting the maximum number of electrons with energies near $E_{0}$, that refrigerate or generate power with Carnot efficiency, and minimizing the number of electrons transmitted in the range $E<E_{0}$ for power generation or in the range $E<\mu_{C}$ and $E>E_{0}$ for refrigeration.

So far we have established the two criteria for high electronic efficiency in thermionic power generators and refrigerators. First, we have shown that the narrower the energy spectrum the higher the electronic efficiency. However, in general a gain in electronic efficiency via this mechanism is obtained at the expense of the power of the device. The second criterion is that the sharper the transition from zero to peak value in the energy spectrum, the higher the electronic efficiency. This second method offers the significant advantage of improving the electronic efficiency without sacrificing power through the use of a narrow filter. The maximum power achievable is also greater with a sharply rising transmission probability if the barrier height is optimized. ${ }^{41}$ In the next section we analyze design considerations for thermionic devices considering both electronic efficiency and power.

\section{DESIGN CONSIDERATIONS FOR ACHIEVING HIGH ELECTRONIC EFFICIENCY IN PRACTICAL DEVICES}

\section{A. Ballistic devices}

Semiconductor-based devices, including superlattices, may be specifically designed to achieve the desired energy spectrum features in $k_{x}$ devices. Filter widths around those required to achieve near-maximum electronic efficiency, as discussed in Sec. V A, may be achieved using a variably spaced superlattice energy filter (VSSEF) as proposed by Summers, Brennan, and Gaylord. ${ }^{42,43}$ Tunneling through a simpler multibarrier structure may also suffice. Similarly, a miniband in the transmission probability for a superlattice might be used as a narrower filter compared with complete transmission above the barrier energy. Quantum dot structures $^{44}$ or NIS devices ${ }^{35}$ can also achieve narrow electron transmission bands and may be used for refrigeration at cryogenic temperatures. Relatively narrow energy electron emission peaks from carbon nanotubes have been reported which may be of use in a vacuum based device. ${ }^{45}$

Since the DOS in the reservoirs fixes the number of electrons available for transport in a certain energy range in ballistic devices, the reduction of power in narrow transmission probability devices, with only modest gains in electronic efficiency, is expected to be undesirable in the presence of phonon heat leaks. It is likely that the best way to simultaneously achieve high electronic efficiency and high power in a ballistic device is to design the structure such that the transmission probability rises sharply from zero to one and remains close to unity beyond this. While the most obvious way to achieve a transmission probability of this nature is to 


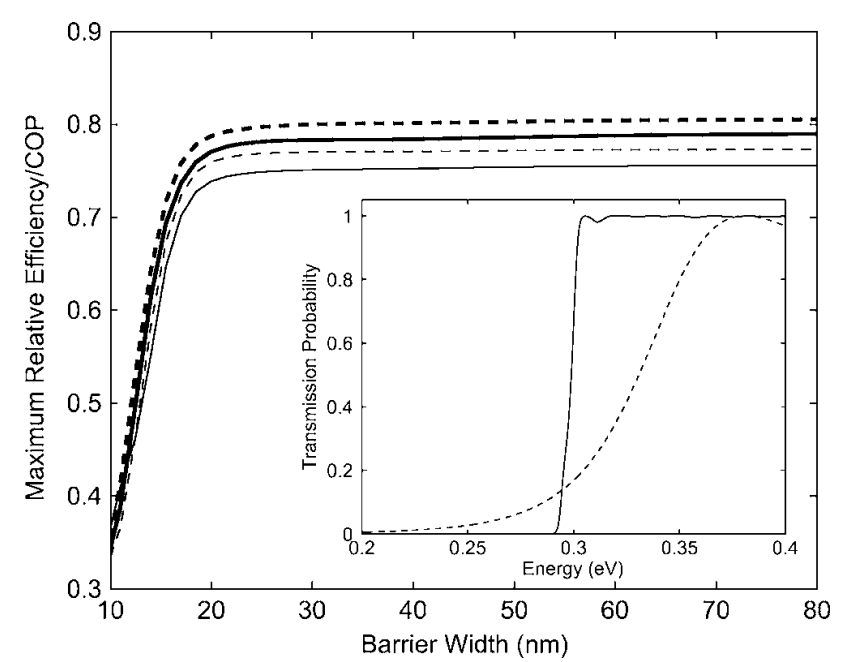

FIG. 7. The relative electronic efficiency and COP of single barrier $k_{r}$ heat engine (thick-dashed line), $k_{x}$ refrigerator (thick-solid line), $k_{x}$ heat engine (thin-dashed line), and $k_{x}$ refrigerator (thinsolid line) devices versus the width of the barrier. $T_{C}=270 \mathrm{~K}, T_{H}$ $=300 \mathrm{~K}, \mu_{C}=0.1 \mathrm{eV}$, barrier height of $0.3 \mathrm{eV}$ and effective mass of $0.067 m_{e}$. (Inset) The transmission probability of a $10 \mathrm{~nm}$ (dashed line) and $100 \mathrm{~nm}$ (solid line) rounded single $0.3 \mathrm{eV}$ barrier systems under no applied bias. Effective mass is $0.067 m_{e}$.

utilize a single barrier with a width as large as possible (but less than the mean free path of electrons) here we show that an array of thin barriers can also be used to engineer a transmission probability that rises sharply from zero to unity.

The transmission probabilities and associated efficiencies/ COPs for single rounded barriers of various widths have been calculated. The transmission probabilities were calculated by obtaining a numerical solution to the timeindependent Schrödinger equation based on Airy functions. ${ }^{31,46}$ Figure 7 (inset) shows the significant difference in sharpness between a $10-\mathrm{nm}$ and $100-\mathrm{nm}$ barrier. We see in Fig. 7, as expected, the wider barriers with sharper transmission probabilities give the highest efficiencies/COPs approaching the maximum value, in this case, at a width of around $35 \mathrm{~nm}$. Beyond this width, there is little to be gained in terms of electronic efficiency, although phonon mediated heat leaks continue to be reduced with increasing barrier width.

From another point of view, wide barriers might be undesirable. Devices with greater interface density may reduce thermal conductivity as a result of interface scattering and phonon miniband formation. ${ }^{47}$ Here we consider a device where multiple barriers are traversed in an electron mean free path. This allows quantum mechanical effects to be utilized to achieve high electronic efficiency using narrow barriers that give low electronic efficiency when used individually.

Multiple narrow barriers over a distance of the order of the electron mean free path may be used to give a transmission probability that is as sharp as if the electrons were traversing a single wide barrier. Figures 8 (a) and 8(b) show the transmission probabilities calculated for two-barrier and eight-barrier systems, respectively, as well as the very gradu-
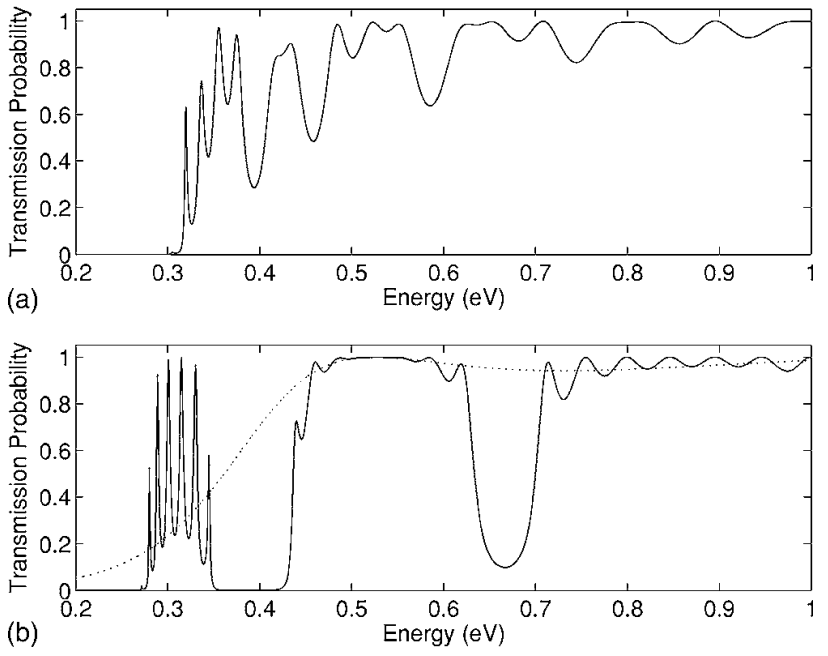

FIG. 8. Transmission probabilities calculated for (a) a two $20 \mathrm{~nm}$ barrier system with $20 \mathrm{~nm}$ well and (b) an eight $5 \mathrm{~nm}$ barrier system with $5 \mathrm{~nm}$ wells (solid) and the transmission probability for a single $5 \mathrm{~nm}$ barrier (dotted) for comparison. $T_{C}=270 \mathrm{~K}, T_{H}$ $=300 \mathrm{~K}, \mu_{C}=0.1 \mathrm{eV}$, barrier height of $0.3 \mathrm{eV}$ and effective mass of $0.067 m_{e}$

ally rising transmission probability for a single $5 \mathrm{~nm}$ barrier for comparison. The efficiencies/COPs achieved are within $3 \%$ of those of a wide single barrier. Thus, high electronic efficiencies may be achieved, while allowing the flexible use of narrower barriers.

We do not expect a dramatic change in dependence of device behavior on the electron energy spectrum as the total length of the device increases beyond an electron mean free path. Since the probability of an electron traveling distance $L$ without suffering a collision is given by $P=\exp (-\lambda / L)$ (Ref. 48) the diffusive and ballistic formalisms, Eqs. (10) and (11), may be combined to show that the electrical current will be given $b^{30}$

$$
J=\iiint q \Delta f\left(D_{r} v_{x}^{r} \zeta P+\frac{\lambda}{L} D_{l} v_{x}^{l}[1-P]\right) d \mathbf{k}
$$

if $L \approx \lambda$. As the overall length of the device becomes significantly greater than the electron mean free path, the ballistic term becomes small so that transport is accurately described using the diffusive formalism.

\section{Diffusive devices}

As was discussed earlier, the energy spectrum in a diffusive device is determined by the product of the local DOS, the velocity squared and the relaxation time. The local DOS of an infinite superlattice may be determined using the Kronig-Penney model. ${ }^{31,49}$ Figure 9 shows the DOS calculated for a many-barrier system and the calculated transmission probability showing their clear relationship. When varying the structure of a device to enhance the electron energy spectrum one must be careful this does not decrease the electron velocity which may reduce current. ${ }^{50}$ Thus the optimization of $D_{l}\left[v_{x}^{l}\right]^{2} \tau$ may be a difficult problem. 


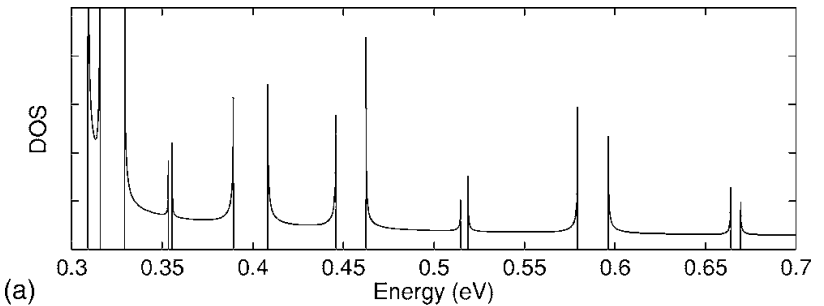

(a)

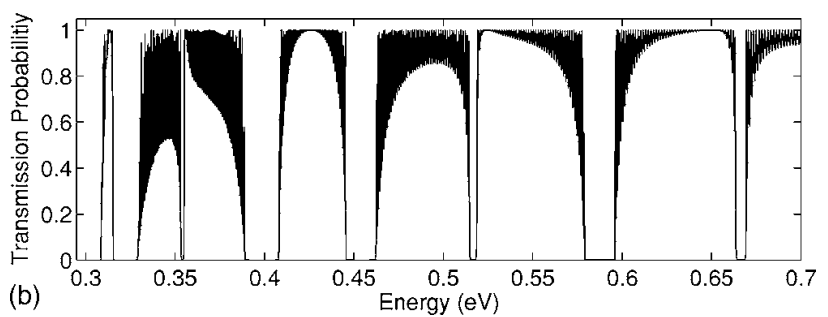

FIG. 9. (a) The local DOS for an infinite series of $20 \mathrm{~nm}$ barriers separated by $20 \mathrm{~nm}$ and (b) the transmission probability for fifty $20 \mathrm{~nm}$ barriers separated by $20 \mathrm{~nm}$ with a barrier height of $0.3 \mathrm{eV}$ and effective mass of $0.067 m_{e}$.

Mahan and Sofo have shown that the ideal transport distribution function for a thermoelectric device may be achieved with a delta function DOS. ${ }^{51}$ Humphrey and Linke showed that the Carnot efficiency may be achieved in thermoelectric devices utilizing a delta function DOS and a graded device structure or inhomogeneous doping. ${ }^{25}$ Their results are analogous to the results presented earlier in this paper where it was shown that the ideal transmission probability for a ballistic device was one that allowed the transmission of only a very narrow energy range of electrons. The results presented in this paper suggest that not only is the width of the energy spectrum important, but also whether it rises rapidly from zero to its maximum value. Hicks and Dresselhaus have pointed out that the magnitude of the DOS at the band edge can be increased by using structures of lower dimensionality, potentially increasing the power factor. ${ }^{17,18}$ We also note that the DOS is sharper for lower dimensional systems compared to bulk materials, which may result in an improved energy spectrum.

\section{EXPERIMENTALLY OBSERVABLE PROPERTIES RELATED TO ELECTRONIC EFFICIENCY}

The presence of phonon heat leaks and contact resistance in solid-state thermionic devices makes direct measurements of the electronic efficiency difficult. Here we discuss experimental properties that may be measured to provide an indication of the shape of the electron energy spectrum and though this, electronic efficiency. Another method that may be useful for determining characteristics of the electron energy spectrum is ballistic electron microscopy/spectroscopy (BEEM/S). ${ }^{52}$

The $I-V$ characteristics of a thermionic device are dependent on the voltage across the barrier system, $V_{B}$. $V_{B}$ may be determined from the supplied voltage, $V_{S}$, and measured current, $I$, as
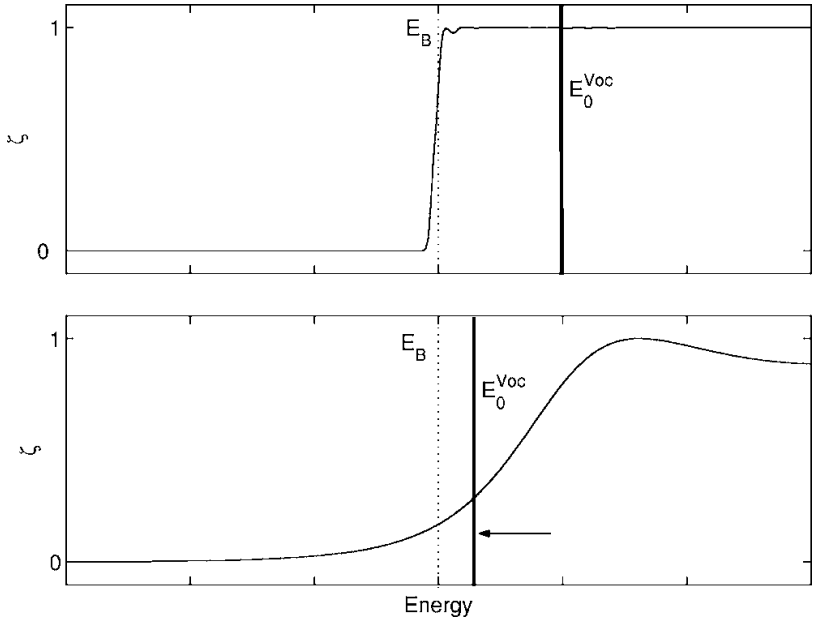

FIG. 10. The transmission probabilities for (a) a wide and (b) a narrow single barrier. $E_{0}^{V_{O C}}$ is indicated by the dashed line and the barrier energy, $E_{B}$ by the dotted line.

$$
V^{B}=V^{S}+I\left(R_{A}+R_{B}\right),
$$

where $R_{A}$ and $R_{B}$ are contact resistances, when the device is generating power. As the bias is increased, the net electrical current decreases and reaches zero at the open circuit voltage $V_{O C}^{B}$, from which the effective Seebeck coefficient may be calculated as

$$
S=\frac{V_{O C}^{B}}{T_{H}-T_{C}} .
$$

The energy-specific equilibrium energy may be calculated at open circuit voltage as

$$
E_{0}^{V_{O C}}=\mu_{C}+V_{O C}^{B} \frac{T_{C}}{T_{H}-T_{H}}=\mu_{C}+S T_{C}
$$

and is linearly related to the Seebeck coefficient. $E_{0}^{V O C}$ is the energy where energy-resolved currents above and below it are equal, giving zero net current. For a sharply rising transmission probability, $E_{0}^{V_{O C}}$ would be positioned as shown in Fig. 10(a) above the barrier energy. If we have another system where electrons with energies lower than the barrier energy are being transmitted without significant change to the high-energy details, for example through decreasing the barrier width, $E_{0}^{V O C}$ is shifted to lower energy as shown in Fig. 10(b). Measuring this relative to a convenient energy, say the barrier energy, $E_{B}$, provides a convenient sharpness indication for the transmission probability

$$
\psi=S T_{C}-\phi
$$

where $\phi=E_{B}-\mu$ is the barrier work function. The "turn-on" energy for a multibarrier system may be shifted to lower energy, in which case, the sharpness indicator should be measured relative to this "effective" barrier height, which might be calculated using the Kronig-Penney model, as discussed previously. The width of the first energy band in a multibarrier system should be sufficiently wide that the distribution function converges in that energy range for this 

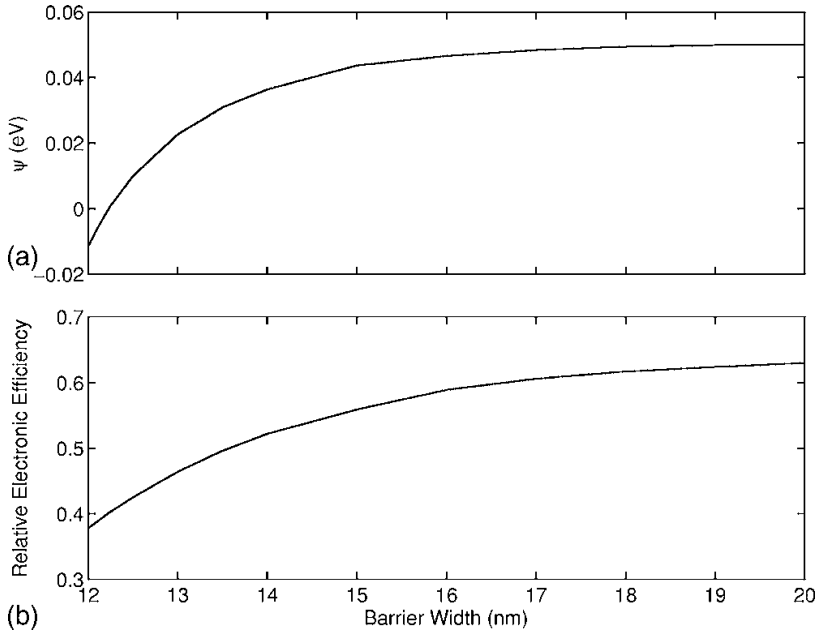

FIG. 11. The (a) maximum relative electronic efficiency and (b) sharpness indicator vs single barrier width. The cold reservoir electrochemical potential has been tuned so that the Seebeck coefficient is constant for each barrier width. $T_{C}=270 \mathrm{~K}, T_{H}=300 \mathrm{~K}$, barrier height of $0.3 \mathrm{eV}$ and effective mass of $0.067 m_{e}$.

technique to be reliable. A higher sharpness indicator is desirable, indicating a sharper transmission probability and therefore higher expected electronic efficiency/COP. The sharpness indicator has the advantage over the Seebeck coefficient of being less dependent on the electrochemical potential/barrier height and more so on the sharpness of the energy spectrum, as shown in Fig. 11. Here, the electrochemical potentials for a number of single barrier transmission probabilities have been varied to give a constant Seebeck coefficient as the barrier width and transmission probability sharpness change. Figure 11 shows the electronic efficiency varies significantly in this example. While the Seebeck coefficient remains constant, the sharpness indicator, $\psi$, increases as the electronic efficiency increases.

\section{CONCLUSIONS}

It has been shown the the nature of the electron-energy spectrum has a significant impact on the performance of thermionic and thermoelectric devices. The limiting efficiency of finite barrier height devices was achieved when electrons of a single energy only are transmitted. While $k_{r}$ devices achieved reversibility when the transmission energy was equal to $E_{0}, k_{x}$ devices did not due to the finite energy spread associated with the two unfiltered degrees of freedom. Electronic efficiency was shown to decrease as the width of the energy spectrum of transmitted electrons increases. Our most important result was that an increase in the sharpness of the rise in electron energy spectrum significantly increases electronic efficiency. Improving the electronic efficiency by increasing the sharpness of the transmission probability may also increase the maximum power. We have shown that sharp transmission probabilities may be achieved using wide single barriers or carefully arranged multiple barriers.

As the equations for electronic efficiency in ballistic and diffusive devices have been shown to have essentially the same dependence upon the electron energy spectrum, the results presented here showing the benefit of sharply rising energy spectra on electronic efficiency and power are expected to be relevant to thermoelectric devices.

Finally, the sharpness indicator, $\psi$, was suggested as an experimental measure providing an indication of the sharpness of the rise in the energy spectrum of a ballistic device and its electronic efficiency and was shown to be superior for this purpose to the Seebeck coefficient alone.

\section{ACKNOWLEDGMENTS}

M.O'D. is supported by the Australian Research Council. T.H. is supported by the Australian Research Council and funding from MURI. The authors acknowledge helpful discussions with Ali Shakouri and Heiner Linke.
*Electronic address: mo15@uow.edu.au

${ }^{1}$ W. Schilichter, Ph.D. thesis, University of Gottingen, 1915.

${ }^{2}$ V. C. Wilson, J. Appl. Phys. 30, 475 (1959).

${ }^{3}$ J. M. Houston, J. Appl. Phys. 30, 481 (1959).

${ }^{4}$ G. D. Mahan, J. Appl. Phys. 76, 4362 (1994).

${ }^{5}$ A. Shakouri and J. E. Bowers, 16th Annual Conference on Thermoelectrics 1997, pp. 636-340.

${ }^{6}$ A. Shakouri and J. E. Bowers, Appl. Phys. Lett. 71, 1234 (1997).

${ }^{7}$ G. D. Mahan and L. M. Woods, Phys. Rev. Lett. 80, 4016 (1998).

${ }^{8}$ G. D. Mahan, J. O. Sofo, and M. Bartkowiak, J. Appl. Phys. 83, 4683 (1998).

${ }^{9}$ A. Shakouri, C. LaBounty, J. Piprek, P. Abraham, and J. E. Bowers, Appl. Phys. Lett. 74, 88 (1999).

${ }^{10}$ C. Labounty, A. Shakouri, P. Abraham, and J. E. Bowers, Proc. SPIE 3950, 69 (2000).

${ }^{11}$ X. Fan, G. Zeng, E. Croke, C. LaBounty, D. Vashaee, A. Shakouri, and J. Bowers, Electron. Lett. 37, 126 (2001).

${ }^{12}$ X. Fan, E. Croke, C. A. Ahn, S. Huxtable, A. Majumdar, and A.
Shakouri, Appl. Phys. Lett. 78, 1580 (2001).

${ }^{13}$ Y. Hishinuma, T. H. Geballe, B. Y. Moyzhes, and T. W. Kenny, Appl. Phys. Lett. 78, 2572 (2001).

${ }^{14}$ Y. Hishinuma, B. Y. Moyzhes, T. H. Geballe, and T. W. Kenny, Appl. Phys. Lett. 81, 4242 (2002).

${ }^{15}$ N. M. Miskovsky and P. H. Cutler, Appl. Phys. Lett. 75, 2147 (1999).

${ }^{16}$ T. S. Fisher and D. G. Walker, J. Heat Transfer 124, 954 (2002).

${ }^{17}$ L. D. Hicks and M. S. Dresselhaus, Phys. Rev. B 47, 12727 (1993).

${ }^{18}$ L. D. Hicks and M. S. Dresselhaus, Phys. Rev. B 47, 16631 (1993).

${ }^{19}$ R. Venkatasubramanian, E. Siivola, T. Colpittes, and B. O'Quinn, Nature (London) 413, 597 (2001).

${ }^{20}$ T. C. Harman, P. J. Taylor, M. P. Walsh, and B. E. LaForge, Science 297, 2229 (2002).

${ }^{21}$ A. A. Balandin, Appl. Phys. Lett. 82, 415 (2003).

${ }^{22}$ D. Vashaee and A. Shakouri, J. Appl. Phys. 95, 1233 (2004). 
${ }^{23}$ D. Vashaee and A. Shakouri, Phys. Rev. Lett. 92, 106103 (2004).

${ }^{24}$ G. D. Mahan, J. Appl. Phys. 70, 4551 (1991).

${ }^{25}$ T. E. Humphrey and H. Linke, Phys. Rev. Lett. 94, 096601 (2005).

${ }^{26}$ X. B. Zhao, X. H. Ji, Y. H. Zhang, J. P. Tu, and X. B. Zhang, Appl. Phys. Lett. 86, 06211 (2005).

${ }^{27}$ G. N. Hatsopoulos and E. P. Syftopoulos, Thermionic Energy Conversion Volume I: Processes and Devices (The MIT Press, London, 1973).

${ }^{28}$ G. S. Nolas and H. J. Goldsmid, J. Appl. Phys. 85, 8 (1999).

${ }^{29}$ C. B. Vining and G. D. Mahan, J. Appl. Phys. 86, 6852 (1999).

${ }^{30}$ M. F. O'Dwyer, T. E. Humphrey, C. Zhang, and R. A. Lewis, J. Appl. Phys. 98, 026108 (2005).

${ }^{31}$ J. H. Davies, The Physics of Low Dimensional Semiconductors-An Introduction (Cambridge University Press, Cambridge, 1998).

${ }^{32}$ M. Nahum, T. M. Eiles, and J. M. Martinis, Appl. Phys. Lett. 65, 3123 (1994).

${ }^{33}$ M. M. Leivo, J. P. Pekola, and D. V. Averin, Appl. Phys. Lett. 52, 5714 (1996)

${ }^{34}$ A. J. Manninen, M. M. Leivo, and J. P. Pekola, Appl. Phys. Lett. 70, 1885 (1997).

${ }^{35}$ H. L. Edwards, Q. Niu, G. A. Georgakis, and A. L. de Lozanne, Phys. Rev. B 52, 5714 (1995).

${ }^{36}$ T. E. Humphrey, R. Newbury, R. P. Taylor, and H. Linke, Phys. Rev. Lett. 89, 116801 (2002).

${ }^{37}$ M. D. Ulrich, P. A. Barnes, and C. B. Vining, J. Appl. Phys. 90, 1625 (2001).

${ }^{38}$ G. J. Snyder and T. S. Ursell, Phys. Rev. Lett. 91, 148301 (2003).

${ }^{39}$ C. Vining, Symposium on Thermoelectric Materials-New Direc- tions and Approaches, edited by T. M. Tritt, MRS Symposia Proceedings No. 278 (Materials Research Society, Pittsburgh, 1997), p. 3.

${ }^{40}$ H. B. Callen, Thermodynamics (Wiley, New York, 1960).

${ }^{41}$ T. E. Humphrey, M. F. O’Dwyer, and H. Linke, J. Phys. D 38, 2051 (2005).

${ }^{42}$ C. J. Summers and K. F. Brennan, Appl. Phys. Lett. 48, 806 (1986).

${ }^{43}$ T. K. Gaylord and K. F. Brennan, Appl. Phys. Lett. 53, 2047 (1988).

${ }^{44}$ T. Bryllert, M. Borgstrom, L. -E. Wernersson, and L. Samuelson, Appl. Phys. Lett. 82, 2655 (2003).

${ }^{45}$ M. J. Fransen, T. L. van Rooy, and P. Kruit, Appl. Surf. Sci. 146, 312 (1999).

${ }^{46}$ K. F. Brennan and C. J. Summers, J. Appl. Phys. 61, 614 (1987).

${ }^{47}$ D. Cahill, W. Ford, K. Goodson, G. Mahan, A. Majumdar, H. Maris, R. Merlin, and S. Phillpot, Jpn. J. Appl. Phys., Part 1 93, 793 (2003).

${ }^{48}$ N. W. Ashcroft and N. D. Mermin, Solid State Physics (Saunders College, Orlando, 1976).

${ }^{49}$ Y. M. Lin and M. S. Dresselhaus, Phys. Rev. B 68, 075304 (2003).

${ }^{50}$ A. Shakouri (personal communication).

${ }^{51}$ D. G. Cahill, W. K. Ford, K. E. Goodson, G. D. Mahan, A. Majumdar, H. J. Maris, R. Merlin, and S. R. Phillpot, J. Appl. Phys. 93, 793 (2003).

${ }^{52}$ K. J. Russell, I. Appelbaum, V. Narayanamurti, M. P. Hanson, and A. C. Gossard, Phys. Rev. B 71, 121311(R) (2005). 